The Egyptian Journal of Hospital Medicine (October 2021) Vol. 85 (1), Page 3272-3276

\title{
Transcutaneous Electric Diaphragmatic Stimulation versus Pilates Method on Lung Functions Post Bariatric Surgery
}

\author{
Wafaa Hussein Borhan ${ }^{1}$, Asem F. Moustafa ${ }^{2}$, Nessrien A. Abd Elrashid ${ }^{1}$, Alaa A.F.A Elnadar*1 \\ ${ }^{1}$ Department of Physical Therapy for Surgery, Faculty of Physical Therapy, Cairo University, Giza, Egypt \\ ${ }^{2}$ Department of Surgery Menoufia University, Faculty of Medicine, Menoufia, Egypt \\ *Corresponding author: Alaa Ali Fathi Ali Elnadar, Mobile: (+20) 01060089494, E-Mail: dralaaali3@gmail.com
}

\begin{abstract}
Background: Bariatric surgeries could alter pulmonary function because of usage of anesthesia as well actual surgical maneuver leading to reduction of residual functional capacity (RFC), precocious closure of small airways and more probability for hypoxemia as well as higher tendency for atelectasis.

Purpose: To evaluate transcutaneous electric diaphragmatic stimulation versus pilates method on lung functions post bariatric surgery.

Subject and Methods: Thirty patients aged 25-40 years old with body mass index (BMI) ranging from $35-60 \mathrm{~kg} / \mathrm{m}^{2}$ underwent sleeve gastrectomy were selected from Outclinics at Menouf General Hospital. They were randomly allocated into two equal groups: Group (A) included 15 participants that received conventional chest physiotherapy plus transcutaneous electric diaphragmatic stimulation as well as medical treatment. While, group (B) included 15 participants that received their conventional chest physiotherapy program, pilates method and medical treatment. Response of lung volumes pre- and post-treatment was recorded.

Results: There was a significant increase of VC and FVC post-treatment in group A \& B compared to pre-treatment values (p > 0.001). Furthermore, increase of VC and FVC in group A (12.08\% \& $12.87 \%$ respectively) also group B (45.19\% \& $52.19 \%$ respectively).

Conclusion: conventional chest physiotherapy in addition to pilates method promote maintenance of pulmonary function. Therefore, it can serve as a preventive character following bariatric surgeries.

Keywords: Bariatric surgery, Electric stimulation, Physiotherapy, Pilates, Respiratory muscle strength, Spirometry.

\section{INTRODUCTION}

Obesity is considered a chronic disorder with complicated etiologies that require variety of treatment options ${ }^{(\mathbf{1})}$. Most of managing pillars were dietetic and physical activity programs plus medicines. Up to date, standard obesity management reported inadequate outcomes clinically, which present actual need for new approaches such as bariatric surgery ${ }^{(2)}$. Unless, anesthetic usage throughout bariatric surgeries could affect pulmonary function, leading to reduction of residual functional capacity, premature closure of small airways and more probability for hypoxemia as well as higher

determining responses to different electrode placement, equipment type, stimuli types, also its parameters, as well various pulmonary function parameters ${ }^{(7)}$. Despite rare clinical trials had examined, electrical stimulation based on expert unanimity lack, plus technical usage problems. However, diaphragmatic electrical stimulation might be quick and safe especially in healthy individuals to increase diaphragmatic strength ${ }^{(\boldsymbol{8})}$. The aim of the study was to evaluate transcutaneous electric diaphragmatic stimulation versus pilates method on lung functions post bariatric surgeries.
\end{abstract} tendency for atelectasis ${ }^{(3)}$. Therefore, obese who already have respiratory abnormalities become worse due to bariatric surgeries, which alter mechanics of respiratory muscle? Such complications explained a result of intraoperative handling, anesthesia, in addition to pain ${ }^{(4)}$. Main accepted explanation was the diaphragm's reflected inhibition that initiated diaphragmatic paresis then restricted pulmonary conduct ${ }^{(5)}$.

Respiratory therapy was recommended in bariatric surgeries for both prophylaxis also management throughout recovery phase. Respiratory therapy was addressed for maintaining as well quickly reorganizing lung function and respiratory musculatures strength ${ }^{(6)}$. Electrotherapy was advised in pulmonary clinical trials on healthy humans. Besides, animal trials that aim for

\section{PATIENTS AND METHODS}

Thirty participants aged 25- 40 years old of both genders, with a BMI of 35- $60 \mathrm{~kg} / \mathrm{m}^{2}$ underwent gastrectomy surgery were selected randomly from Outclinics at Menouf General Hospital, then allocated into two equal groups: Group (A): Fifteen patients underwent sleeve gastrectomy surgery received traditional chest physiotherapy (diaphragmatic respiratory, deep inhalation, also respiratory exercises associated with shoulder flexion and extension of the upper extremities, assisted cough, circulatory exercises, and early ambulation), plus transcutaneous electric diaphragmatic stimulation in addition to their medical treatment. 
Group (B): Fifteen patients underwent sleeve gastrectomy surgery received traditional chest physiotherapy program (diaphragmatic respiratory, deep inhalation exercises, also respiratory exercises associated with shoulder flexion and extension of the upper extremities, assisted cough, circulatory exercises, and early ambulation), plus pilates method, in addition to their medical treatment.

\section{Ethical approval:}

The present study was approved by the Ethical Committee, Faculty of Physical Therapy, Cairo University. Research Ethics Committee and quality control approvals were held. Present study was explained in details to each participant before obtaining their informed written consent. Quality control of screening, handling of data and verification of adherence to protocols were done on a regular basis by trial coordinator. This work has been carried out in accordance with The Code of Ethics of the World Medical Association (Declaration of Helsinki) for studies involving humans.

Inclusion Criteria: Age ranged between 25-40 years, all patients` BMI were $35-60 \mathrm{~kg} / \mathrm{m}^{2}$, who underwent gastric sleeve, and all patients were free of any acute or chronic pulmonary disease and have the capacity to perform the protocol evaluation tests properly.

Exclusion Criteria: Cardiac disorders, chronic pulmonary disorders, previous other abdominal surgeries, and previous or present smoking history.

The procedure of the study was divided into two categories:

\section{1-Measurement procedures:}

The evaluation was conducted before and after the study protocol.

0) Procedures of spirometer:

Numerous techniques for performing spirometry were addressed. For FVC and FEV1, the patient took a deep breath, as large as possible, and then blows out as hard and fast as possible kept going until no air left. PEF was obtained from the FEV1 and FVC maneuver. For VC, the patient took a deep breath, as large as possible, and then blows steadily for as long as possible until no air left. Nose clips were essential for $\mathrm{VC}$ as air could leak out due to low flow. The IVC maneuver was performed at the end of FVC/VC (depending on device used) by taking a deep, fast breath back in after breathing all the way out. Encouragement made a big differ, so do not be afraid to raise your voice for encouragement by end of the maneuver to keep blowing until no more air comes out and volume time trace reached a plateau; $50 \mathrm{Ml}$ being exhaled in 2 seconds ${ }^{(9)}$.

\section{2-Therapeutic procedures:}

a) Transcutaneous electrical stimulation procedures: Two rubber electrodes' pair were used; one was located over parasternal region near to xiphoid process, while the other pair over corresponding region to diaphragmatic motor points at $6^{\text {th }} \& 7^{\text {th }}$ intercostal spaces in line with right and left front armpits. Electrodes were fixed by micropore bandage, where skin was previously cleaned with alcohol. The placement of the electrodes was by hand-touch, while participant was in dorsal recumbent position with the bed head raised by $30^{\circ}$, the knees were semi-inflected, the feet were supported, the arms were stretched alongside the body and the head was on the pillow. After marking electrode site, the point of placement was confirmed through visual muscle contraction observation, when the electric current was applied later. The session extended to thirty minutes ${ }^{(\mathbf{1 0})}$.

b) Procedures of Pilates method: performed in different positions, as well previous documented to have low impact on various body' structures, particularly musculatures, joints, also ligaments, particularly on lumbosacral region. Thus, any populations could gain benefits ${ }^{(11)}$. The force center was made up of i) abdominal musculatures; rectus abdominis, internal also external oblique, plus transversus abdominis, ii) the gluteus and also, iii) paraspinal lumbar region. It was responsible for body static and dynamic stabilization ${ }^{(\mathbf{1 2})}$. Thus, force center formed a structure that is responsible for spinal support as well internal organs; stabilizing the trunk; maintaining correct posture, with respect to lower energy expenditure ${ }^{(\mathbf{1 3})}$. Moreover, injury susceptibilities were controlled. The force center should remain in contraction throughout execution of all exercises. In line with Joseph Pilates concerning complete fitness, it was recommended for breathing, indicating that all exercises should be associated with adequate continuous breathing rhythm (12).

In the Pilates method, respiratory style accentuated costal breathing, where ribs should ascend then descend, as well laterally extended to posterior across breathing flow. The transverse muscle was specified to prevent abdominal distension, provided greater diaphragmatic support that promote lower ribs motion, also better diaphragmatic excursion. While at expiratory stage, contraction of transversus abdominis muscles was associated with multifidus muscles and pelvic floor contraction ${ }^{(\mathbf{1 4})}$.

c) Physical therapy program for both groups:

Step 1: Diaphragmatic breathing training, flow or volume incentive spirometry; 3 sets, 5 deep breaths repetitions.

Step 2: Airway clearance techniques; huffing and/ or coughing. 
Step 3: Circulation; foot- ankle pumping and hip- knee bending ten repetitions per hour.

Step 4: Thoracic expansion training; patient remain in long sitting/ high sitting over bed side.

Step 5: Mobilization: a) Sitting out of the bed; one hour twice daily. B) Walking; three times per day. C) Stair climbing just before discharge ${ }^{(\mathbf{1 5})}$.

\section{Statistical analysis}

Descriptive statistical analysis also unpaired t-test were conducted for comparison of study population characteristics. Normal data distribution was checked using Shapiro-Walk test. Levine's test was used for examining variances of homogeneity in between groups. Unpaired t-test was conducted to compare the mean values of VC and FVC between groups. Paired t-test was used for comparison between pre- and post-treatment per groups. The level of significance was set at $p \leq 0.05$. All statistical analysis was conducted through the statistical package for social trials (SPSS) version 25 for windows (IBM SPSS, Chicago, IL, USA).

\section{RESULTS}

Table (1) showed the subject characteristics of the group A and B. There was no significant difference between both groups in the mean age, weight, height and BMI ( $p>0.05)$. Also, there was no significant difference in sex distribution between groups $(\mathrm{p}=0.71)$.

Table (1): Comparison of subject characteristics between group

A and B

\begin{tabular}{|c|c|c|c|c|c|}
\hline & \multicolumn{2}{|c|}{ Mean \pm SD } & \multirow[b]{2}{*}{ MD } & \multirow[b]{2}{*}{$\begin{array}{c}\text { t- } \\
\text { value }\end{array}$} & \multirow[b]{2}{*}{$\begin{array}{c}\text { p- } \\
\text { value }\end{array}$} \\
\hline & Group A & $\begin{array}{c}\text { Group } \\
\text { B }\end{array}$ & & & \\
\hline Age (years) & $\begin{array}{l}34 \pm \\
4.97\end{array}$ & $\begin{array}{l}32 \pm \\
5.12\end{array}$ & 2 & 1.08 & 0.28 \\
\hline Weight (kg) & $\begin{array}{c}140.66 \pm \\
20.78\end{array}$ & \begin{tabular}{|r|}
140.33 \\
\pm 16.33 \\
\end{tabular} & 0.33 & 0.04 & 0.96 \\
\hline Height (cm) & $\begin{array}{c}167.6 \pm \\
8.77\end{array}$ & $\begin{array}{l}167.26 \\
\pm 7.16\end{array}$ & 0.34 & 0.11 & 0.91 \\
\hline BMI $\left(\mathrm{kg} / \mathrm{m}^{2}\right)$ & $\begin{array}{c}49.88 \pm \\
5.94 \\
\end{array}$ & $\begin{array}{c}50.17 \pm \\
6.01\end{array}$ & -0.29 & -0.13 & 0.89 \\
\hline Males/Females & $9 / 6$ & $9 / 6$ & & & \\
\hline
\end{tabular}

Mean; SD, Standard deviation; MD, Mean difference; $\mathrm{p}$ value, Probability value.

\section{Effect of treatment on VC and FVC: \\ Within group' comparison:}

There was a significant increase in VC and FVC posttreatment in group $\mathrm{A}$ and $\mathrm{B}$ compared to that of pretreatment $(\mathrm{p}<0.001)$. The percent of increase in $\mathrm{VC}$ and FVC in group A were $12.08 \%$ and $12.87 \%$ respectively and that for group B were $45.19 \%$ and $52.19 \%$ respectively (Table $2 \&$ figure 1 ).

\section{Between groups' comparison:}

There was no significant difference in the $\mathrm{VC}$ and FVC between both groups pre-treatment $(\mathrm{p}>0.05)$. Comparison between groups $\mathrm{A}$ and $\mathrm{B}$ post-treatment revealed a significant increase in VC and FVC of group B compared to that of group A $(\mathrm{p}<0.001)$ (Table 2 and figure $1)$.

Table (2): Mean VC and FVC pre and post treatment of the group $\mathrm{A}$ and $\mathrm{B}$

\begin{tabular}{|c|c|c|c|c|c|}
\hline & Group A & Group B & \multirow[t]{2}{*}{ MD } & \multirow[t]{2}{*}{ t- value } & \multirow[b]{2}{*}{ p value } \\
\hline & $\begin{array}{c}\text { Mean } \pm \\
\text { SD }\end{array}$ & $\begin{array}{c}\text { Mean } \pm \\
\text { SD }\end{array}$ & & & \\
\hline \multicolumn{6}{|l|}{ VC (L) } \\
\hline $\begin{array}{l}\text { Pre } \\
\text { treatment }\end{array}$ & $\begin{array}{r}1.82 \pm \\
0.39\end{array}$ & $1.77 \pm 0.42$ & 0.05 & 0.32 & 0.74 \\
\hline $\begin{array}{l}\text { Post } \\
\text { treatment }\end{array}$ & $\begin{array}{r}2.04 \pm \\
0.37\end{array}$ & $2.57 \pm 0.42$ & -0.53 & -3.63 & 0.001 \\
\hline MD & -0.22 & -0.8 & & & \\
\hline$\%$ of change & 12.08 & 45.19 & & & \\
\hline \multirow[t]{2}{*}{$\mathrm{t}$ - value } & -6.64 & -12.37 & & & \\
\hline & $p=0.001$ & $p=0.001$ & & & \\
\hline \multicolumn{6}{|l|}{ FVC (L) } \\
\hline $\begin{array}{l}\text { Pre- } \\
\text { treatment }\end{array}$ & $2.02 \pm 0.7$ & $2.05 \pm 0.6$ & -0.03 & -0.12 & 0.89 \\
\hline $\begin{array}{l}\text { Post } \\
\text { treatment }\end{array}$ & $\begin{array}{r}2.28 \pm \\
0.68 \\
\end{array}$ & $3.12 \pm 0.51$ & -0.84 & -3.76 & 0.001 \\
\hline MD & -0.26 & -1.07 & & & \\
\hline$\%$ of & 12.87 & 52.19 & & & \\
\hline $\mathrm{t}$ - value & -5.26 & -11.36 & & & \\
\hline & $p=0.001$ & $p=0.001$ & & & \\
\hline
\end{tabular}

Mean; SD, standard deviation; MD, mean difference; $p$-value, probability value

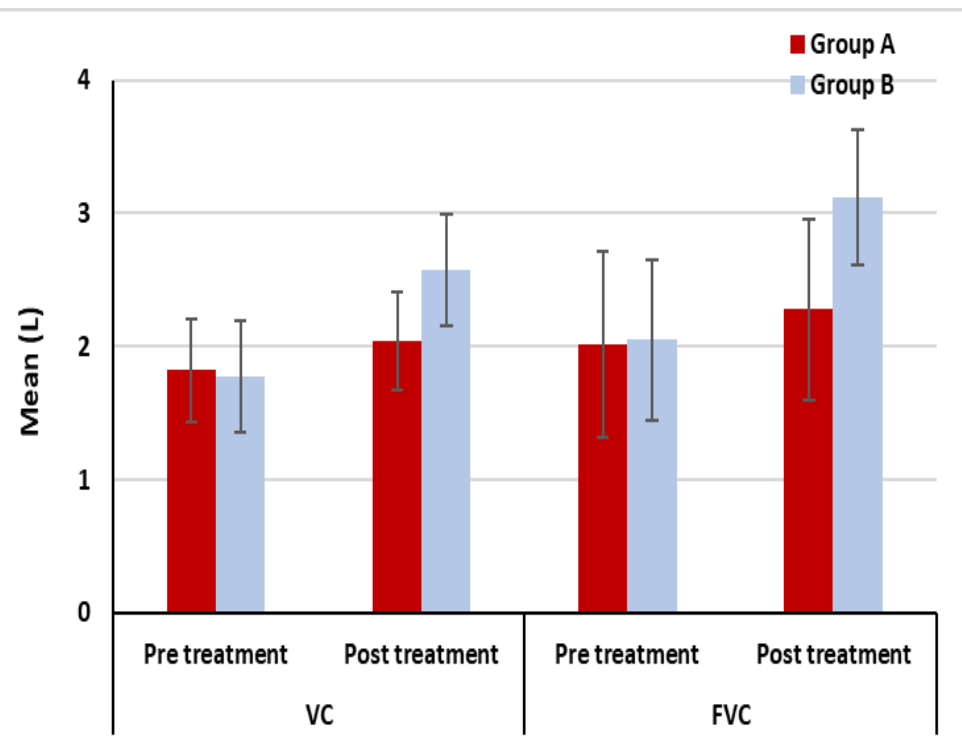

Figure (2): Mean VC and FVC pre- and post-treatment of the group $\mathrm{A}$ and $\mathrm{B}$ 


\section{DISCUSSION}

There was lack of evidence of spirometry differences between study populations that gives more reliability to present study inclusion criteria, as well to population homogeneity. Because of no significant differences in between groups also for various evaluating measures, both treatments protocol could be contributed for prevention of pulmonary function parameters regression. Those were normally quoted in medical literature ${ }^{(10,11)}$.

Rubinstein et al. ${ }^{(16)}$ found that limitations in respiratory flow ranged between 50-75\% of vital capacity in obese persons. Although Rubinstein and his colleagues either did not exclude or even decline to report that they did exclude participants with co-morbidities and/or respiratory dysfunctions. Some previous clinical trials including Luce ${ }^{(17)}$ have recorded expiratory reserve volume, functional residual capacity (FRC) and tidal volume (TV) decrease, which have led to altered ventilation also perfusion even shunts, resulting in subsequent hypoxemia especially in obese patients with pulmonary dysfunction.

Current study reported a significant increase in VC \& FVC post-treatment in both groups compared to that pre-ones $(\mathrm{p}>0.001)$ with percentages in group $\mathrm{A}$ were $12.08 \%$ \& $12.87 \%$ respectively and also for group B were $45.19 \%$ \& $52.19 \%$ respectively. Our results came in agreement with Rigatto ${ }^{(18)}$ and Barros et al. ${ }^{(19)}$ findings subsequent to such surgeries as well two weeks later on hypo-caloric diet. They reported a drop in respiratory musculature` strength associated with lower lean mass as well led to reduction of diaphragm thickness. In addition, Rovina et al. ${ }^{(20)}$ and Joris et al. ${ }^{\text {(21) }}$ ensured diaphragmatic dysfunction that explained by reflexive suppression of phrenic nerve, which explained lowered maximal expiratory pressure (MEP) through postoperative period after any open abdominal surgeries by that it was due to abdominal musculatures being sliced, which make it difficult to create expiratory pressures. In the same line, Toledo and Garcia ${ }^{(22)}$ on the $14^{\text {th }}$ postoperative day, had discovered that MIP levels were extremely near to that obtained at preoperative period, unless Toledo and Garcia did not care for MEP maintenance in whom underwent physiotherapy treatment.

Our clinical trial reinforced importance of respiratory physiotherapy for bariatric surgeries, which comes in line with what concluded by Rovina et al. ${ }^{(20)}$ who did not use respiratory physiotherapy and detected diminished MIP values postoperatively. As well, Paisani et $\boldsymbol{a l} .{ }^{(11)}$ ensured diminished MIP also MEP values than those recorded preoperative at $5^{\text {th }}$ postoperative day for bariatric surgeries. Toledo and Garcia ${ }^{(22)}$ had reported that impairment of respiratory musculatures` strength by two weeks and one month postoperative after discharged, as well the time delay and that chest physiotherapy was designed to prevent such negative side effects including pain and/ or diaphragmatic paresis.

According to medical literature, importance of adding chest physical therapy as integral part post bariatric surgeries were supported by Eli $\boldsymbol{e t}$ al. ${ }^{(23)}$ for maintenance of inhalation muscles` strength. As well, conventional chest physiotherapy was recorded to be similar to conventional chest physiotherapy (CCP) if combined with transcutaneous electric diaphragmatic stimulation (TEDS). More and above, it was recorded that expiratory muscular strength of CCP participants were not maintained by two and four weeks postoperatively, unless was maintained in CCP+TEDS participants. Thus, CCP+TEDS could be advised as essential prophylactic management for bariatric surgeries through postoperative phase. Furthermore, Cancelliero et al. ${ }^{(24)}$ conducted twice sessions per week along six weeks on twenty-one healthy women examining two diaphragmatic transcutaneous electrical stimulation maneuvers. They ensured that maximum expiratory pressure (MEP) was improved by 44.7- $60.9 \%$, respectively, while maximum inspiratory pressure (MIP) was improved by 32.9- 63.2\%, respectively. Based on pretrial MIP values, respiratory muscle strength improvements could be ensured. As well, overlapping of stimulation zone gained increase in both values. In addition, applied electrical stimuli create a large electric field that was sufficient to stimulate surrounding musculatures. Another study conducted by Santos $\boldsymbol{e t}$ al. ${ }^{(25)}$ who performed thirty minutes session daily along two weeks on fourteen elders as well TEDS participated and then reported that MEP \& MIP also tidal volume had increased. MIP was modulated from 42.1412.67 to 55.7112.84, MEP from 63.2119.18 to 83.5720.52, as well tidal volume was improved from 411.4379 .79 to $453.24164 .93 \mathrm{~mL}$.

In same line with our study, Debastiani and Aroca ${ }^{(26)}$ had ensured that TEDS' usage for elders for ten sessions extended for twenty minutes had provided $25 \%$ improvements on maximum inspiratory pressure.

Why Pilates considered more effective than transcutaneous diaphragmatic electrical stimulation?

Leivne et al. ${ }^{\text {(27) }}$ concluded that Pilates concentrate on improving activities leading to strengthening of minor muscles also subsequent strengthening of major ones could be gained as well. Keays $\boldsymbol{e t} \boldsymbol{a l} .{ }^{(\mathbf{2 8})}$ had examined Pilates for breast cancer women with limited mobility, and concluded that Pilates might be introduced as effectual and safe therapeutic modality for regaining upper extremities function and mobility. On the other hand, numerous studies had recorded Pilates profoundly adversely affect stabilometric variables ${ }^{(29)}$.

Erfani et al. ${ }^{(30)}$ found that a Pilate's routine training for male knee osteoarthritis could be used to 
improve function and quality of life in a safe and effective maneuver. Rydeard et al. ${ }^{\left({ }^{(3)}\right)}$ had evaluated a four weeks Pilates-based training on low back pain (LBP), around specific musculatures that help to stabilize the lumbopelvic region and reported significant improvement compared to control group. They added that modified Pilates program can help people with LBP in reducing pain and disability.

Finally, the findings of this study implied that both traditional respiratory therapy also pilates, plus traditional physiotherapy in addition to transcutaneous electric diaphragmatic stimulation could improve pulmonary function parameters maintenance as well as a preventive role following bariatric surgeries. In addition it ensured that Pilates training usage in conjunction with other therapeutic modalities can ensure functional performance gains. The study was limited to physical as well psychological status of participants that might influence evaluation and treatment efficacy.

\section{CONCLUSION}

It can be concluded that both traditional chest physiotherapy also pilates approach assist to maintain pulmonary function as well serve as an important preventive measure after bariatric surgeries.

\section{Financial support and sponsorship: Nil.}

\section{Conflict of interest: Nil.}

\section{REFERENCES}

1. Biener A, Cawley J, Meyerhoefer C (2017): The impact of obesity on medical care costs and labor market outcomes in the US. Clin Chem., 64: 108-117.

2. Segal A, Fandiño J (2002): Indications \& contra-indications for carrying out bariatric surgery. Rev Bras Psiquiatr., 24: 68-72.

3. Eichenberger A, Proietti S, Wicky S et al. (2002): Morbid obesity and postoperative pulmonary atelectasis: an underestimated problem. Anesth Analg., 95: 1788-92.

4. Olsén M, Lönroth H, Bake B (2007): Effects of breathing exercises on breathing patterns in obese and non-obese subjects. Clinical Physiology, 19: 251-7.

5. Chiavegato D, Jardim J, Faresin S (2003): Functional respiratory alterations in laparoscopic cholesystectomy. J Bras Pneumol., 26: 69-76.

6. Olsén M, Hahn I, Nordgren S et al. (2007): Randomized controlled trial of prophylactic chest physiotherapy in major abdominal surgery. Br J Surg., 84: 1535-8.

7. Geddes L, Voorhees W, Babbs C (2009): Electroventilation. Amer Journ Emer Med., 3: 337-9.

8. Criner G, Travaline J, Holt G et al. (2009): Variability of electrophrenic diaphragm twitch stimulation over time in normal subjects. Respir Physiol., 118: 39- 47.

9. Miller M, Hankinson J, Brusasco V et al. (2005): Standardisation of spirometry. Eur Respir J., 26: 319-338.

10. Geddes L, Voorhees W, Bourland C et al. (1990): Optimum stimulus frequency for contracting the inspiratory muscle with chestsurface electrodes to produce artificial respiration. Rev Ann Biomed Eng., 18: 103-8.

11. Paisani D, Chiavegatto L, Faresin S (2005): Volumes, pulmonary capacities and respiratory muscle strength in postoperative gastroplasty. J Bras Pneum., 31: 125-32.
13. Rodrigues B (2006): Método Pilates: uma nova proposta em reabilitação física. eFisioterapiea Web site. http://www.efisioterapia.net/ descargas/ pdfs/ pilates.pdf.

14. Gómez V, García O, Ejercicio Y (2009): Pilates durante el embarazo. Educ Fis Deportes, 14 (136): 236-242.

15.Pires D, Sá C (2005): Pilates notas sobre aspectos históricos, princípios, técnicas e aplicações. Educ Fis Deportes, 10 (91): 157162.

16. Boussuges A, Gole Y, Blanc $P$ (2009): Diaphragmatic motion studied by m-mode ultrasonography: methods, reproducibility, and normal values. Chest, 135: 391- 400 .

17. Rubisntein I, Zamel N, DuBarry L et al. (1990): Airflow limitation in morbidly obese nonsmoking men. Ann Intern Med., 112: 828-32.

18. Luce J (1980): Respiratory Complication of Obesity. Chest, 78: 626-31.

19. Rigatto A (2005): Ventilatory performance in obesity. Saúde em Rev., 17: 57-62.

20. Barros S, Moura L, Carvalho M (2003): Correlation between maximum respiratory pressures and the nutritional status of patients with lung disease. Rev Bras Nutr Clin., 18: 123-9.

21. Rovina N, Bouros D, Tzanakis $\mathrm{N}$ et al. (1996): Effects of laparoscopic cholesystectomy on global respiratory muscle strength. Am J Respir Crit Care Med., 153: 458-61.

22. Joris J, Hinque V, Laurent $P$ et al. (1998): Pulmonary function and pain after gastroplasty performed via laparotomy or laparoscipy in morbidly obese patients. BR J Anaesth., 80: 283-8.

23. Toledo R, Garcia R (2005): Complicações pulmonares e alterações na mecânica respiratória e na ventilometria em pós-operatório de gastroplastia / Pulmonary complications and alterations in the respiratory mechanics and ventilometry in post-operative gastroplasty. Reabilitar., 29: 32-40.

24. Eli F, Daniela I, Marcela B et al. (2009): Effects of Chest Physiotherapy on the Respiratory Function of Postoperative Gastroplasty Patients. Clinics (Sao Paulo), 64 (7): 683-689.

25. Cancelliero K, Ike D, Sampaio L et al. (2012): Transcutaneous electrical diaphragmatic stimulation (TEDS) for the respiratory muscle strengthening: randomized and controlled clinical study. Fisioter Pesqui., 19 (4): 303-8.

26. Santos L, Borg J, Daister J et al. (2013): Diaphragmatic effects of the transcutaneous electrical stimulation on the pulmonary function in elderly. Rev Bras Geriatr Gerontol., 16 (3): 495-502.

27. Debastiani M, Aroca J (2009): Effectiveness of diaphragmatic neuromuscular electrical stimulation on inspiratory muscle strength in institutionalized elderly. Proceedings of the III Uniamérica Physical Therapy Seminar, 9: 25-26.

28. Levine B, Kaplanek B, Scafura D et al. (2007): Rehabilitation after total hip and knee arthroplasty: a new regimen using Pilates training. Bulletin of the NYU Hospital for Joint Diseases, 65 (2): 120-125.

29. Keays K, Harris S, Lucyshyn J et al. (2006): Effects of Pilates exercises on shoulder range of motion, pain, mood and upper extremity function in women living with breast cancer: a pilot study. Phys Ther., 88 (4): 494-510.

30. Patti A, Bianco A, Paoli A et al. (2016): Pain Perception and Stabilometric Parameters in People With Chronic Low Back Pain After a Pilates Exercise Program. Medicine, 95(2):1-7.

31. Erfani M, Mehrabian H, Shojaedin S et al. (2012): Effects of Pilates exercise on knee osteoarthritis in elderly male athletes. J Res Rehabil Sci., 7: 571-579.

32. Rydeard R, Andrew L, Drew S (2006): Pilates-based therapeutic exercise: effect on subjects with nonspecific chronic low back pain and functional disability: a randomized controlled trial. Journal of Orthopedic \& Sports Physical Therapy, 36: 472-484. 